\title{
Regulation of Acylcoenzyme A \\ Cholesterol Acyltransferase and \\ 3-Hydroxy-3-Methylglutaryl Coenzyme A \\ Reductase Activity by Lipoproteins in the Intestine of Parabiont Rats
}

Bryce H. Purdy and F. Jeffrey Field

Department of Internal Medicine, University of Iowa, Iowa City, Iowa 52242

bstract. Parabiont rats were used to study the regulation of intestinal cholesterol synthesis (3-hydroxy3-methylglutaryl coenzyme A [HMG-CoA] reductase activity) and esterification (acylcoenzyme A/cholesterol acyltransferase [ACAT] activity) by lipoproteins and micellar cholesterol. The parabiont rat model offers a unique way to observe changes in cholesterol metabolism in the intestine, independently of luminal factors. In one group of six surgically joined pairs, one animal was fed rat chow and $0.1 \%$ propylthiouracil (PTU). Its joined mate was fed rat chow, $0.1 \%$ PTU, 5\% lard, and $0.3 \%$ taurocholic acid. In another group of five pairs, one rat was fed rat chow, $0.1 \%$ PTU, 5\% lard, 0.3\% taurocholic acid, plus $1 \%$ cholesterol. Its joined mate was fed the same diet except the cholesterol was deleted.

Serum cholesterol changes were equal between members of a given pair, attesting to their common circulation. The administration of PTU itself caused a significant elevation of serum cholesterol. When one parabiont ingested cholesterol, serum cholesterol concentrations increased significantly for both pair members compared with control pairs not ingesting cholesterol.

Hepatic and intestinal HMG-CoA reductase activities were significantly decreased in rats fed the diet containing cholesterol. ACAT activities in both organs were markedly increased. This supports previous data that suggest that dietary or luminal cholesterol affects both HMG-CoA reductase and ACAT activity in the small intestine. Moreover, in rats that were hypercholesterolemic but not ingesting dietary cholesterol, hepatic and intestinal reductase activities were decreased and ACAT activities

Received for publication 27 November 1983 and in revised form 12 April 1984.

J. Clin. Invest.

(c) The American Society for Clinical Investigation, Inc.

0021-9738/84/08/0351/07 \$1.00

Volume 74, August 1984, 351-357 were increased compared with the control animals. Intestinal microsomal cholesterol content was increased only in rats fed cholesterol. No changes in intestinal microsomal cholesterol were observed in the other animals. The data suggest that intestinal HMG-CoA reductase and ACAT activities are regulated by plasma lipoproteins independently of luminal factors. This nonluminal regulatory effect occurs without a measurable contribution to the intestinal microsomal cholesterol pool.

\section{Introduction}

The understanding of the regulation of cholesterol metabolism in the small intestine is incomplete. From the early work of Dietschy and Siperstein (1), it was postulated that cholesterol synthesis in the intestine, unlike the liver, was not regulated by feedback inhibition by dietary cholesterol. Shefer et al. (2) measured intestinal 3-hydroxy-3-methylglutaryl coenzyme A (HMG$\mathrm{CoA})^{1}$ reductase in rats fed $2 \%$ cholesterol. They also observed no differences in intestinal reductase activity compared with that of rats fed normal chow. However, in intestinal cells obtained from these cholesterol-fed rats, the cholesterol content was not increased. When the intestinal cellular content was increased by feeding rats $2 \%$ cholesterol in combination with either sodium taurocholate or chenodeoxycholate, intestinal HMG-CoA reductase activity was significantly decreased. Other investigators have since documented the regulation of intestinal cholesterol synthesis by luminal cholesterol (3-5). In cultured intestinal explants, cholesterol has been shown to inhibit cholesterol synthesis (6) and stimulate cholesterol esterification (7).

Bile acids also regulate intestinal cholesterol metabolism. The binding of bile acids in the lumen of the intestine by resins (3), or the surgical diversion of bile from the intestine $(3,8)$ causes a marked stimulation of cholesterol synthesis and a decrease in cholesterol esterification in the intestine (9). The latter observation is associated with a significant loss of microsomal cholesterol throughout the small intestine (9). Hormones have

1. Abbreviations used in this paper: ACAT, acylcoenzyme A/cholesterol acyltransferase; HMG-CoA, 3-hydroxy-3-methylglutaryl coenzyme A; PTU, propylthiouracil. 
also been shown to regulate cholesterol synthesis in cultured intestinal explants $(10,11)$.

There is evidence now to suggest that the enterocyte can use circulating cholesterol in times of severe plasma cholesterol deficiency. Anderson and Dietschy $(12,13)$ and, more recently, Panini et al. (14) have observed a marked increase in intestinal cholesterol synthesis in rats treated with the drug 4-aminopyrazolo $(3,4-d)$ pyrimidine, which causes a profound hypocholesterolemia. Both groups suggested that this increase in intestinal cholesterol synthesis was secondary to a decrease in the delivery of plasma cholesterol to the enterocyte. In the study of Panini et al. (14) it was observed that the stimulation of cholesterol synthesis occurred not only in intestinal crypt cells but also in absorptive cells. This suggests that lipoprotein cholesterol regulates cholesterol metabolism in cells of the intestine that are fully differentiated and whose major function is to absorb exogenous nutrients.

In short-term culture of canine ileum, Gebhard and Cooper (6) did not observe the regulation of HMG-CoA reductase by lipoproteins. Later, however, in intestinal cultures extended for $24 \mathrm{~h}$, lipoproteins were found to be potent regulators of cholesterol synthesis (15). These findings agree with previous reports that suggest that freshly isolated tissue or cells may not express lipoprotein receptors until cultured in lipoprotein-deficient medium for an extended period $(16,17)$. Recently, lipoprotein receptors have been identified on isolated intestinal cells (18). Gebhard and Prigge (19) showed in vivo that in isolated intestinal Thiry-Vella loops, intestinal HMG-CoA reductase was regulated by plasma lipoproteins independently of luminal factors. In contrast to these findings, Stange and Dietschy (20) recently observed no changes in low density lipoprotein (LDL) uptake in the rat intestine despite major experimental changes in the rate of intestinal sterol synthesis.

The present study was therefore undertaken to reexamine the influence of circulating cholesterol on intestinal cholesterol synthesis (HMG-CoA reductase activity) and cholesterol esterification (acylcoenzyme A/cholesterol acyltransferase [ACAT] activity).

Surgically joined parabiont rats were used. This technique ensures vascular exchange between two animals while maintaining separate digestive tracts. Thus, enterocytes of a parabiont pair are exposed to the same lipoprotein concentrations but maintain discrete luminal environments. The control of luminal and vascular cholesterol concentrations permitted independent assessment of in vivo lipoprotein cholesterol regulation on HMGCoA reductase and ACAT activities in the intestine. Our results support the hypothesis that cholesterol metabolism in the small intestine is regulated by lipoprotein cholesterol.

\section{Methods}

Chemicals. $\left[1-{ }^{14} \mathrm{C}\right]$ oleoyl coenzyme A, $\left[1,2-{ }^{3} \mathrm{H}(\mathrm{N})\right]$ cholesterol, $\left[5-{ }^{3} \mathrm{H}\right]-$ mevalonolactone, and [glutaryl-3- $-{ }^{14} \mathrm{C}$ ] HMG-CoA were purchased from New England Nuclear (Boston, MA). Oleoyl-coenzyme A, cholesterol, propylthiouracil (PTU), sodium taurocholate, and trypsin inhibitor were from Sigma Chemical Co., St. Louis, MO). HMG-CoA was purchased from P-L Biochemicals Inc. (Milwaukee, WI). 3 $\alpha$-Hydroxysteroid dehydrogenase (EC 1:1:1:50) was obtained from Worthington Biochemical Corp. (Freehold, NJ). All other chemicals were reagent grade.

Animals and diet. Inbred male littermate rats (F344 Sprague-Dawley; Fisher Scientific Co., Madison, WI) weighing $115 \pm 30 \mathrm{~g}$ were obtained for parabiosis. The parabiotic union was created by a modified technique of Warren et al. (21). The rats to be joined were anesthetized with Nembutal (Abbott Pharmaceuticals Inc., North Chicago, IL). After the skin was shaved and cleansed with alcohol, a cutaneous incision was made from the ileac crest to the base of the ear in each animal. Skin flaps were made dorsally and ventrally by blunt dissection. Excess skin was excised and the adjacent ventral skin flaps were joined by skin clips. The adjacent scapulae were sutured securely together with nonabsorbable suture. Initially, the ileac crests were also sutured together, but we found that this was unnecessary for a good union. The dorsal skin flaps were then pinned by clips. The parabiont rats were permitted a 4-wk recovery period. They were allowed free movement and access to rat chow (5012; Ralston Purina Co., St. Louis, MO) and water ad lib. One rat of a joined pair failed to thrive during the recovery period and the pair was killed. No adverse behavior was observed in the other 11 pairs.

In a pilot study which used five surgically joined pairs, and in one pair from the present experimental group, the formation of the parabiontic union was evaluated. ${ }^{51} \mathrm{Cr}$-labeled erythrocytes $(2,500 \mathrm{cpm})$ were injected into the tail vein of one of the animals. After the injection, both members were monitored with scintillation detectors for the amount of radioactivity present (22). A lead shield placed between the two animals prevented cross-contamination of radiation. If the level of radioactivity became equal in the two animals, the vascular union was considered patent. In all five pairs of the pilot study, this equilibration occurred within $1 \mathrm{~h}$ after injection. In the pair from the present experimental group, $4 \mathrm{wk}$ after surgical union, equilibration of the isotope occurred within $20 \mathrm{~min}$ after the injection.

Before the test diets were started, blood for cholesterol determination was obtained by tail vein section under light ether anesthesia. Because each rat of a joined pair was to receive a different diet, an apparatus to preclude crossover feeding was necessary. A feeding chamber was built for each pair, as depicted in Fig. 1. The diameter of each cylinder was such that it allowed only a single rat to enter. Access to the food required the animal to move along the cylinder. For a rat of a joined pair to feed, it needed the cooperation of its mate to enter the feeding device together. This cooperative behavior was readily learned within the first $12 \mathrm{~h}$ of exposure to the apparatus. The common channel terminated two-thirds down the length of the cylinders. Thus, their sutured adjacent scapulae prevented exiting through the other end.

The following powdered diets were prepared: diet A, rat chow (Ralston Purina Co.) and 0.1\% PTU; diet B, rat chow and 0.1\% PTU, 5\% lard, $0.3 \%$ sodium taurocholate; and diet C, rat chow and $0.1 \%$ PTU, $5 \%$ lard, $0.3 \%$ sodium taurocholate, $1 \%$ cholesterol (23).

The animals were given equal amounts of their diet each morning in a disposable aluminum cup. No systematic attempt was made to ensure equal caloric intake. By the next morning, however, all the chow in the cups had been eaten. They were allowed free access to water. At the end of a 6-wk feeding period, the animals were anesthetized with pentobarbital and their peritoneal cavities were opened. All animals were killed between 8 and $9 \mathrm{AM}$. Blood was obtained by aortic puncture, the entire small intestine was excised, and a wedge of liver was resected. Sera were frozen for analysis. Enzyme analysis was done on the same day as sacrifice. Microsomal membranes were frozen at $-80^{\circ} \mathrm{C}$ for later determination of cholesterol content. 


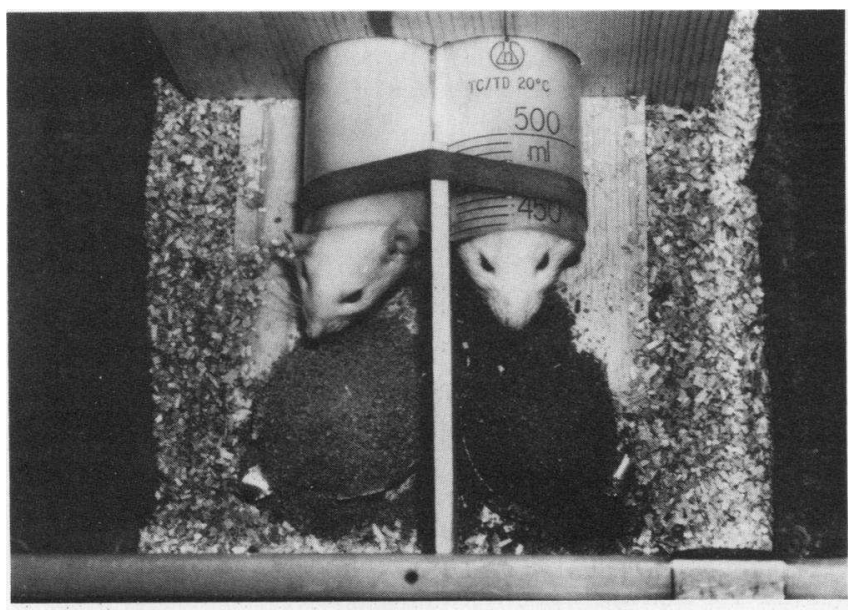

Figure 1. Parabiont rats in feeding chamber. To prevent crossover feeding of the joined pairs, the rats were fed in the device shown above. To obtain food, the parabiont rats have to enter the device together. The small diameter of the cylinders allows only a single rat to enter each cylinder. Joint movement down the cylinders permits the individual animals to feed. A partition between the cylinders and the dietary cups prevents animals from eating the other diet.

Biliary drainage. In a separate group of 18 inbred male littermate rats (F344 Sprague-Dawley) weighing $150-160 \mathrm{~g}$, biliary drainage was performed. The animals were first divided into three dietary groups and given diet A, B, or $\mathrm{C}$ ad lib. for $6 \mathrm{wk}$. At the end of this period, the bile duct was cannulated with PE-10 tubing in each animal, under light ether anesthesia. After 10-15 min had elapsed to ensure a constant flow of bile, the bile was collected for 1 to $2 \mathrm{~h}$, depending upon the rate of flow. At the end of the collection, blood was taken by aortic puncture and the animals were killed. Biliary drainage was done between 0800 and 1230 hours.

Preparation of microsomes. The entire small intestine from each animal was divided into three equal segments: segment 1 (proximal), segment 2 (middle), and segment 3 (distal). After the lumens were thoroughly rinsed with cold normal saline, the segments were opened and the intestinal mucosa was gently scraped into Dounce homogenizers containing a buffer of $0.1 \mathrm{M}$ sucrose, $0.05 \mathrm{M} \mathrm{KCl}, 0.04 \mathrm{M} \mathrm{KH}_{2} \mathrm{PO}_{4}$, $0.03 \mathrm{M}$ EDTA, and $0.1 \mathrm{mg} / \mathrm{ml}$ trypsin inhibitor, $\mathrm{pH}$ 7.4. Microsomes were prepared as previously described (9). Hepatic microsomes were prepared in the same manner as intestinal microsomes, except the buffer did not contain trypsin inhibitor.

Enzyme assays. ACAT activity was measured in hepatic and intestinal microsomes as previously described for the intestine (9). The specific activity of oleoyl-CoA was $14,370 \mathrm{dpm} / \mathrm{nmol}$. HMG-CoA reductase activity was measured as described previously (24). The specific activity of HMG-CoA was $24,260 \mathrm{dpm} / \mathrm{nmol}$.

Chemical analysis. Serum cholesterol was measured by gas chromatography according to the method of Ishikawa (25). Lipids were extracted from bile and microsomes with chloroform/methanol (2:1, $\mathrm{vol} / \mathrm{vol}$ ). Free and total cholesterol were determined by gas chromatography with cholestane as an internal standard to determine recoveries. Cholesterol from Applied Science was used as a standard. Chromatography was performed on a gas chromatograph (5840 A; Hewlett Packard Co., McMinnville Div., McMinnville, OR) with a 3-ft glass column containing 3\% OV-1 on $80 / 100$ Supelcoport (Supelco, Inc., Bellefonte, PA). Total bile acids in bile were determined with $3 \alpha$-hydroxysteroid dehydrogenase as described by Turley and Dietschy (26). Protein was determined according to the method of Lowry et al. (27).

Statistical analysis. The paired and unpaired $t$ tests were used to determine probability values.

\section{Results}

Parabiont rat model. It is recognized that rats made hypothyroid by being fed PTU markedly diminish their food intake. In our experimental design, all rats were made hypothyroid by being fed equivalent amounts of PTU. Their metabolic state and caloric intake, therefore, were similar. During the 4-wk period between surgical union of the pairs and the start of the test diets, the rats in both parabiont groups gained weight at a steady and equal rate. The weights of the parabiont rats, however, remained relatively constant after the test diets containing PTU were started. None of the pairs lost weight during the 6-wk dietary period. As shown in Table I, there were no significant differences between the weights of the two groups of parabiont rats prestudy or at sacrifice. This suggests that the caloric intake among the pairs was similar despite the dietary differences.

Table I. Weights* of Parabiont Rats

\begin{tabular}{llll}
\hline Parabiont rats & Pair number & Prestudy & At sacrifice \\
\hline Group I & 1 & 170 & 278 \\
& 2 & 178 & 280 \\
& 3 & 198 & 302 \\
& 4 & 210 & 290 \\
& 5 & 205 & 309 \\
& 6 & 262 & 286 \\
Group II & Average & $204 \pm 13$ & $291 \pm 5$ \\
& 7 & 166 & \\
& 8 & 175 & 272 \\
& 9 & 310 & 376 \\
& 10 & 238 & 310 \\
& 11 & 240 & 318 \\
& Average & $226 \pm 26$ & $305 \pm 22$ \\
\hline
\end{tabular}

Rats 1 of Group I were fed rat chow plus 0.1\% PTU (diet A). Rats 2 of group I were fed rat chow, $0.1 \%$ PTU, $5 \%$ lard, and $0.3 \%$ taurocholic acid (diet B). Rats 1 of group II were fed the same diet as rats 2 of Group I (diet B). Rats 2 of group II were fed rat chow, $0.1 \%$ PTU, 5\% lard, 0.3\% taurocholic acid, and 1\% cholesterol (diet C). Prestudy weights were determined at the time of surgical union. After surgery, the pairs were allowed a 4-wk recovery period during which time they were allowed food and water ad lib. The test diets were then fed for $6 \mathrm{wk}$. The animals were weighed just before they were killed.

* Grams. 
Serum cholesterol. Table II shows the serum cholesterol concentrations in the two groups of parabiont rats. In group I, one rat was fed rat chow and $0.1 \%$ PTU (diet $\mathrm{A}$ ). Its joined partner received chow, $0.1 \%$ PTU, 5\% lard, and $0.3 \%$ taurocholate (diet B). In group II, one rat received Diet B and its mate received Diet B plus $1 \%$ cholesterol (diet C). The cholesterol concentrations prestudy and at sacrifice were not significantly different between the individual animals of the joined pairs in both groups. This is evidence that the parabiont rats share a common circulation. Serum cholesterol concentrations at sacrifice in group I were increased compared with prestudy concentrations. Feeding PTU alone to rats has been shown to be associated with an increase in serum cholesterol levels (28). In eight individual rats that were fed chow and $0.1 \%$ PTU, mean cholesterol levels increased from 55 to $98 \mathrm{mgdl}^{-1}$ (data not shown). This correlates closely with changes observed in group I parabionts and suggests that the changes in serum cholesterol concentrations observed in this group were a secondary effect of the abnormal hormonal state and were not due to the surgical procedure or to the dietary lard and bile acid.

At sacrifice, serum cholesterol levels were significantly higher in group II parabiont rats than in group I. The increase in serum cholesterol concentration in parabiont rats that were fed diet B in group II is caused by the ingestion of cholesterol (diet C) by their paired mates. The gastrointestinal tracts of animals fed diet B in group II assimilate the same amount of cholesterol as those of animals in group I that receive diet $B$.

HMG-CoA reductase activity. An increase in serum cholesterol concentration should affect the rate of cholesterol synthesis and esterification (29). Table III shows the effect of dietary modification on the rate-controlling enzyme of cholesterol synthesis, microsomal HMG-CoA reductase, in the parabiont rats. There was significant variability of reductase activity that occurred between rats within the same dietary group. Nevertheless,

Table II. Serum Cholesterol Concentrations* in Parabiont Rats

\begin{tabular}{ccc}
\hline Parabiont rats & Prestudy & At sacrifice \\
\hline Group I & & \\
Diet A & $37 \pm 2$ & $91 \pm 5 \ddagger$ \\
Diet B & $36 \pm 1$ & $87 \pm 3 \ddagger$ \\
Group II & & \\
Diet B & $40 \pm 6$ & $192 \pm 23 \ddagger \S$ \\
Diet C & $43 \pm 6$ & $229 \pm 14 \ddagger \S$ \\
\hline
\end{tabular}

Blood was taken from the tail vein of each rat for cholesterol determinations prior to the start of diet A, B, or C (see Table I). These were termed prestudy values. At the end of 6 wk on the test diets, blood was taken by aortic puncture for cholesterol analysis. These values were at sacrifice.

* mg/dl, mean $\pm \mathrm{SE}$.

$\ddagger P<0.001$ vs. prestudy, determined by the paired $t$ test. $\S P<0.001$ vs. group I, at sacrifice, determined by the unpaired $t$ test.
Table III. Hepatic and Intestinal Microsomal HMG-CoA Reductase Activities* in Parabiont Rats

\begin{tabular}{rcccc}
\hline $\begin{array}{l}\text { Parabiont } \\
\text { rats }\end{array}$ & Liver & Intestine 1 & Intestine 2 & Intestine 3 \\
\hline $\begin{array}{r}\text { Group I } \\
\text { Diet A }\end{array}$ & $27 \pm 9$ & $53 \pm 10$ & $48 \pm 14$ & $59 \pm 18$ \\
Diet B & $14 \pm 6$ & $61 \pm 22$ & $58 \pm 16$ & $44 \pm 11$ \\
Group II & & & & \\
Diet B & ND & $27 \pm 3$ & $22 \pm 4 \pi$ & $18 \pm 5 \pi$ \\
Diet C & $3 \pm 1 \ddagger$ & $15 \pm 5 \S$ & $15 \pm 3 \pi$ & $20 \pm 5$ \\
\hline
\end{tabular}

Diets A, B, and C (see Table I) were fed for 6 wk. The animals were killed and a wedge of liver and the entire small intestine were removed. The intestine was divided into three equal segments: intestine 1 , proximal; intestine 2, middle; and intestine 3, distal. Microsomes were prepared from the liver wedge and the three segments of small bowel. Enzyme assays were done on the day of sacrifice. $P$ values were determined by the unpaired $t$ test. ND, not detected.

* pmol mevalonate formed/mg per min, mean \pm SE.

$\ddagger P<0.01$ vs. group $\mathrm{I}$, diets $\mathrm{A}$ and $\mathrm{B}$.

$\S P<0.01$ vs. group I, $\operatorname{diet} A$.

I $P<0.05$ vs. group I, diet $\mathrm{B}$.

" $P<0.05$ vs. group I, diet A.

significant differences were observed. The well described effect of feedback regulation of hepatic HMG-CoA reductase by dietary cholesterol is evident. Parabiont rats in group II had significantly decreased hepatic reductase activities. This supports the observation that hepatic cholesterol synthesis is very sensitive to regulation by lipoproteins.

The activity of HMG-CoA reductase in the intestine was also regulated by the dietary modifications. The individual rats in group II that were fed cholesterol (diet C) had decreased HMG-CoA reductase activities in each segment of small bowel, compared with parabiont rats in group I. This effect of luminal cholesterol and bile salt on intestinal reductase activity has been noted before (2). However, rats that were not fed cholesterol but had significantly elevated serum cholesterol levels (diet B, group II) had significantly depressed intestinal HMG-CoA reductase activity in segments 2 and 3, compared with reductase activity in rats fed the same diet in the absence of circulating hypercholesterolemia (diet B, group I). This supports the observations of Gebhard and Prigge, who suggested that plasma lipoproteins regulate intestinal cholesterol synthesis independently of luminal cholesterol (19).

ACAT activity. Table IV shows the results of ACAT activity in microsomes prepared from the liver and small intestine of the parabiont rats. In group II rats, hepatic ACAT activity responded dramatically to the ingestion of cholesterol. ACAT activity in these livers increased five- to sixfold compared with hepatic ACAT activity from group I rats. Intestinal ACAT activity was also affected significantly by dietary manipulation in the parabiont rats. In rats receiving cholesterol in their diet (diet 
Table IV. Hepatic and Intestinal Microsomal ACAT Activities* in Parabiont Rats

\begin{tabular}{rllll}
\hline $\begin{array}{l}\text { Parabiont } \\
\text { rats }\end{array}$ & Liver & Intestine 1 & Intestine 2 & Intestine 3 \\
\hline $\begin{array}{rlll}\text { Group I } \\
\text { Diet A }\end{array}$ & $223 \pm 50$ & $420 \pm 86$ & $510 \pm 88$ & $239 \pm 70$ \\
Diet B & $214 \pm 42 \ddagger$ & $291 \pm 115 \ddagger$ & $538 \pm 78$ & $101 \pm 40 \ddagger$ \\
Group II & & & & \\
Diet B & $1,181 \pm 231 \S$ & $660 \pm 66$ & $854 \pm 197$ & $360 \pm 119$ \\
Diet C & $1,428 \pm 206 \ddagger \S \pi$ & $875 \pm 206 \$ \pi$ & $1,350 \pm 88 \ddagger \S \pi$ & $704 \pm 140 \ddagger \S \pi$ \\
\hline
\end{tabular}

Diets A, B, and C (see Table I) were fed for 6 wk. The animals were killed and a wedge of liver and the entire small intestine were removed. The intestine was divided into three equal segments: intestine 1, proximal; intestine 2, middle, and intestine 3, distal. Microsomes were prepared from the liver wedge and the three segments of small bowel. Enzyme assays were done on the day of sacrifice. $P$ values were determined by the unpaired $t$ test.

* pmol cholesteryl oleate formed/mg per min, mean \pm SE.

$\ddagger P<0.05$ vs. group II, diet B.

$\S P<0.02$ vs. group I, diet $A$.

I $P<0.005$ vs. group $\mathrm{I}$, diet $\mathrm{B}$.

C, group II), ACAT activity was markedly increased in every segment of small bowel, compared with intestinal ACAT activity in group I rats. In the middle and distal segments of intestine from rats fed diet C, ACAT activity was significantly higher than the activity observed in their paired mates fed diet $B$. This supports our previous data (9) and data from others $(30,31)$ which indicate that luminal or dietary cholesterol increases intestinal ACAT activity. Moreover, in rats that did not ingest cholesterol but had increased circulating cholesterol levels (diet B, group II), ACAT activity in the intestine was higher than ACAT activity observed in the intestine from rats fed the same diet but that had less circulating cholesterol (diet B, group I). Significance was reached in the proximal and distal segment, but the trend in the mid-small bowel was apparent.

Microsomal cholesterol. It is felt that the microsomal free cholesterol pool plays a role in the regulation of HMG-CoA reductase and ACAT activity $(32,33)$. Table $\mathrm{V}$ shows the microsomal cholesterol content from intestine and liver of the parabiont rats. Hepatic microsomal cholesterol content from rats in group II was significantly higher than in group I. Intestinal microsomal cholesterol content was increased in rats fed the diet containing cholesterol (diet C). Microsomal cholesterol content in parabiont rats fed diet B in group II, however, was no different from that in rats in group $I$.

\section{Discussion}

Unlike most cells within the body that must rely upon either the de novo synthesis of free cholesterol or the binding and uptake of lipoproteins to maintain sterol balance, the enterocyte has three mechanisms for obtaining cholesterol. The small in-
Table V. Microsomal Cholesterol Content* in Liver and Intestine from Parabiont Rats

\begin{tabular}{rllll}
\hline $\begin{array}{l}\text { Parabiont } \\
\text { rats }\end{array}$ & Liver & Intestine 1 & Intestine 2 & Intestine 3 \\
\hline $\begin{array}{r}\text { Group I } \\
\text { Diet A }\end{array}$ & $14 \pm 2 \ddagger$ & $25 \pm 4$ & $32 \pm 2$ & $36 \pm 6$ \\
Diet B & $14 \pm 2 \ddagger$ & $29 \pm 5$ & $33 \pm 6$ & $36 \pm 5$ \\
Group II & & & & \\
Diet B & $31 \pm 3$ & $30 \pm 3$ & $28 \pm 3$ & $47 \pm 7$ \\
Diet C & $37 \pm 6$ & $39 \pm 3 \pi$ & $44 \pm 5$ \$ा & $50 \pm 7$ \\
\hline
\end{tabular}

Microsomes were prepared from the liver and the three segments of small intestine. Membrane cholesterol was analyzed by gas-liquid chromatography as described in Methods. $P$ values were determined by the unpaired $t$ test.

* $\mu \mathrm{g}$ cholesterol $/ \mathrm{mg}$ protein, mean $\pm \mathrm{SE}$.

$\ddagger P<0.001$ vs. group II, diets B and C.

$\S P<0.05$ vs. group II, diet B.

II $P<0.05$ vs. group I, diet A.

testine ranks second only to the liver in de novo synthesis of cholesterol (1). Its regulation has been extensively studied $(1-3,6,8,10,11)$. Dietary cholesterol, which is absorbed by the small intestine, is also available for use by the enterocyte. No other cell in the body has this capacity. Finally, like other cells, the enterocyte is in contact with plasma lipoproteins and, at least in theory, could obtain cholesterol by this means. Our study supports the observations of others $(14,15,19)$ who suggest that lipoproteins regulate intestinal HMG-CoA reductase. This is the first report, however, that shows that intestinal ACAT is also regulated by this mechanism.

The parabiont rat model offers advantages over the isolated intestinal Thiry-Vella loop. In our model, the small intestine of each animal maintains its normal absorptive function throughout the experimental period. Therefore, mucosal atrophy secondary to deprivation of luminal nutrients, as observed with the isolated loop (34), is not a problem. The intestinal tract of the control animal in a joined pair, however, still absorbs cholesterol from bile. An increase in biliary cholesterol excretion secondary to the dietary modifications might affect HMG-CoA reductase and ACAT activity in the gut. There is good evidence in the literature that suggests that biliary cholesterol concentration does not depend upon hepatic cholesterol synthesis, the size of the hepatic cholesteryl ester pool, or the hepatic uptake of chylomicron cholesterol $(2,35)$. However, because these studies were done with euthyroid rats, we repeated these previous experiments under experimental conditions similar to those that exist in our present parabiont model. A separate group of 18 rats (F344 Sprague-Dawley), weighing slightly more than the parabiont rats to facilitate bile duct cannulation, were fed either $\operatorname{diet} \mathrm{A}, \mathrm{B}$, or $\mathrm{C}$ ad lib. for $6 \mathrm{wk}$. At the end of this period, plasma cholesterol levels were $51 \pm 1,63 \pm 3$, and $208 \pm 18 \mathrm{mgdl}^{-1}$, respectively. As expected, biliary cholesterol output was unchanged 
by the diets: $0.46 \pm 0.04,0.57 \pm 0.04$, and $0.54 \pm 0.03 \mu \mathrm{mol} / \mathrm{kg}$ per $h$, respectively. Bile acid output, however, was significantly increased in the rats fed diet B compared with those fed diet A, $98.8 \pm 12.9$ vs. $58.1 \pm 6.5 \mu \mathrm{mol} / \mathrm{kg}$ per $\mathrm{h}$, but not with those fed diet $\mathrm{C}, 55.1 \pm 3.7 \mu \mathrm{mol} / \mathrm{kg}$ per $\mathrm{h}$. Therefore, the changes observed in ACAT and HMG-CoA reductase activities in the intestines of the parabionts cannot be secondary to changes in biliary cholesterol.

Initial hepatic HMG-CoA reductase activities were low in parabiont rats in group I (Table III). There are two reasons that account for this. All animals were killed when hepatic cholesterol synthesis was at its nadir $(36,37)$. The abnormally low metabolic state of the animals, which in turn is associated with a decrease in food consumption, can also lead to a decrease in hepatic reductase activity $(38,39)$. The dramatic inhibition of hepatic HMG-CoA reductase activity which occurred in the animals that were fed cholesterol, however, suggests that the axis for feedback regulation of hepatic HMG-CoA reductase was intact despite the abnormal hormonal or nutritional status of the animals.

HMG-CoA reductase activity was inhibited in the intestine of rats fed the atherogenic diet (Table III, diet C). This supports the data of Shefer et al. (2), which showed that feeding cholesterol and a bile salt to rats inhibits cholesterol synthesis in the intestine. Intestinal ACAT activity was also markedly increased in rats fed cholesterol (Table IV, diet C). This supports our previous work in rabbits (9), which demonstrated the regulation of intestinal ACAT by luminal cholesterol. It is of interest that the most marked changes in HMG-CoA reductase and ACAT activities were observed in the intestine of these animals which were fed cholesterol, compared with their joined mates and with rats of the other group. This suggests that dietary cholesterol plays a major role in the regulation of cholesterol homeostasis in the small intestine.

In the intestine of rats that were hypercholesterolemic but not ingesting cholesterol (group II, diet B), HMG-CoA reductase activity was suppressed and ACAT activity was stimulated, compared with their appropriate controls (Tables III and IV). This suggests strongly that the small intestine can take up and degrade plasma lipoproteins. The subsequent regulatory events that occur in the enterocyte are the same as those that occur in fibroblasts and smooth muscle cells (see reference 29 for a review). The released free cholesterol in the cell down-regulates HMG-CoA reductase and stimulates ACAT activity. We observed an increase in intestinal microsomal cholesterol content in rats fed cholesterol (Table V) but not in the animals fed the other diets. Despite the absence of gross measurable changes in microsomal free cholesterol, HMG-CoA reductase and ACAT activities were changed in these animals (group II, diet B). It has been suggested that there are separate small free-cholesterol pools in the microsomal membrane that are near HMG-CoA reductase and ACAT. Regulation of these enzymes has been shown to occur without any gross changes in microsomal free cholesterol content (32). It can be readily observed from our data, however, that when microsomal free cholesterol content does increase, there is a more dramatic effect on the activities of both HMG-CoA reductase and ACAT than when no measurable change occurs.

In resistant species such as the rat and dog, extremes in dietary protocols are essential to induce significant hypercholesterolemia (23). The diet used in this study causes a significant increase in apoprotein E-containing lipoproteins such as $\mathrm{HDL}_{\mathrm{c}}$ and $\beta$-very low density lipoproteins. These abnormal lipoproteins induced by dietary cholesterol have a high affinity for apoprotein B, E (LDL) receptors, as well as for $E$ receptors $(40,41)$. Therefore, they are potent regulators of cholesterol metabolism in cells containing these receptors. The regulation of cholesterol metabolism in the intestine by lipoproteins may require the generation of these apoprotein E-rich lipoproteins; as Stange and Dietschy (20) recently observed, the regulation of cholesterol synthesis and LDL receptor activity in the rat intestine were independent.

The contribution that lipoproteins make to the cholesterol pool of the enterocyte is not known. In two separate studies in which LDL was modified so that its catabolic pathway could be followed, it was discovered that $\sim 6-8 \%$ of the LDL injected into rats was catabolized by the small intestine $(42,43)$. Under normal circumstances, therefore, it appears that the need for lipoprotein cholesterol by the enterocyte is not great (20). This seems appropriate for a cell with direct access to dietary (micellar) cholesterol.

\section{Acknowledgments}

We thank F. R. Kirchner and J. W. Osborne for their guidance and help in preparing the parabiont rats. We are grateful to Ms. Ella Albright, Ms. Kathy Funk, and Ms. Julie Ostrem for typing the manuscript.

This work was supported in part by grants 81-G-14 from the American Heart Association, Iowa Affiliate; Atherosclerosis Specialized Center of Research, HL-14230 from the National Heart, Lung and Blood Institute; and AM 29706 from the National Institute of Arthritis, Metabolism and Digestive Diseases.

\section{References}

1. Dietschy, J. M., and M. D. Siperstein. 1967. Effect of cholesterol feeding and fasting on sterol synthesis in seventeen tissues of the rat. $J$. Lipid Res. 8:97-104.

2. Shefer, S., S. Hauser, V. Lapar, and E. H. Mosbach, 1973. Regulatory effects of dietary sterols and bile acids on rat intestinal HMG CoA reductase. J. Lipid Res. 14:400-405.

3. Dietschy, J. M. 1968. The role of bile salts in controlling the rate of intestinal cholesterogenesis. J. Clin. Invest. 47:286-300.

4. Wilson, J. D. 1972. The relation between cholesterol absorption and cholesterol synthesis in the baboon. J. Clin. Invest. 51:1450-1458.

5. Swann, A., and M. D. Siperstein. 1972. Distribution of cholesterol feedback control in the guinea pig. J. Clin. Invest. 51:95a. (Abstr.)

6. Gebhard, R. L., and A. D. Cooper. 1978. Regulation of cholesterol synthesis in cultured canine intestinal mucosa. J. Biol. Chem. 253:27902796.

7. Field, F. J., A. D. Cooper, and S. K. Erickson. 1982. Regulation of rabbit intestinal acyl coenzyme A-cholesterol acyltransferase in vivo and in vitro. Gastroenterology. 83:873-880. 
8. Dietschy, J. M., and J. D. Wilson. 1968. Cholesterol synthesis in the squirrel monkey: relative rates of synthesis in various tissues and mechanisms of control. J. Clin. Invest. 47:166-174.

9. Field, F. J., and R. G. Salome. 1982. Effect of dietary fat saturation, cholesterol, and cholestyramine on acyl-CoA:cholesterol acyltransferase activity in rabbit intestinal microsomes. Biochim. Biophys. Acta. 712:557570.

10. Goodman, M. W., W. R. Prigge, and R. L. Gebhard. 1981. Hormonal regulation of canine intestinal cholesterol synthesis. Am. J. Physiol. 240:G274-G280.

11. Stange, E. F., G. Preclik, A. Schneider, E. Seiffer, and H. Ditschuneit. 1981. Hormonal regulation of 3-hydroxy-3-methylglutaryl coenzyme A reductase and alkaline phosphatase in cultured intestinal mucosa. Biochim. Biophys. Acta. 678:202-206.

12. Andersen, J. M., and J. M. Dietschy. 1976. Cholesterogenesis: derepression in extrahepatic tissues with 4-aminopyrazolo [3,4-d] pyrimidine. Science (Wash. DC). 193:903-905.

13. Andersen, J. M., and J. M. Dietschy. 1977. Regulation of sterol synthesis in 15 tissues of rat. J. Biol. Chem. 252:3652-3659.

14. Panini, S. R., G. Lehrer, D. H. Rogers, and H. Rudney. 1979. Distribution of 3-hydroxy-3-methylglutaryl coenzyme A reductase and alkaline phosphatase activities in isolated ileal epithelial cells of fed, fasted, cholestyramine-fed, and 4-aminopyrazolo [3,4-d] pyrimidinetreated rats. J. Lipid Res. 20:879-889.

15. Stange, E. F., M. Alavi, A. Schneider, G. Preclik, and H. Ditschuneit. 1980. Lipoprotein regulation of 3-hydroxy-3-methylglutaryl coenzyme A reductase in cultured intestinal mucosa. Biochim. Biophys. Acta. 620:520-527.

16. Reichl, D., A. Postiglione, and M. B. Myant. 1976. Uptake and catabolism of low density lipoprotein by human lymphocytes. Nature (Lond.). 260:634-635.

17. Ho, Y. K., M. S. Brown, D. W. Bilheimer, and J. L. Goldstein. 1976. Regulation of low density lipoprotein receptor activity in freshly isolated human lymphocytes. J. Clin. Invest. 58:1465-1474.

18. Suzuki, N., N. Fidge, P. Nestel, and J. Yin. 1983. Interaction of serum lipoproteins with the intestine. Evidence for specific high density lipoprotein-binding sites on isolated rat intestinal mucosal cells. J. Lipid Res. 24:253-264.

19. Gebhard, R. L., and W. F. Prigge. 1981. In vivo regulation of canine intestinal 3-hydroxy-3-methylglutaryl coenzyme $A$ reductase by cholesterol, lipoprotein, and fatty acids. J. Lipid Res. 22:1111-1118.

20. Stange, E. F., and J. M. Dietschy. 1983. Cholesterol synthesis and low density lipoprotein uptake are regulated independently in rat small intestine epithelium. Proc. Natl. Acad. Sci. USA. 80:5739-5743.

21. Warren, S., E. E. Hurst, Jr., C. E. Brown, and R. N. Chute. 1972. Anastomotic sarcoma of irradiated parabiont rats. Cancer Res. 32:983-987.

22. Kirchner, F. R., and J. W. Osborne. 1978. Failure to find a humoral factor which influences the compensatory response after resection of the rat small bowel. Cell Tissue Kinet. 11:227-234.

23. Mahley, R. W., and K. S. Holcombe. 1977. Alterations of the plasma lipoproteins and apoproteins following cholesterol feeding in the rat. J. Lipid Res. 18:314-324.

24. Field, F. J., S. K. Erickson, M. A. Shrewsbury, and A. D. Cooper. 1982. 3-Hydroxy-3-methylglutaryl coenzyme A reductase from rat intestine: subcellular localization and in vitro regulation. J. Lipid Res. 23:105-113.

25. Ishikawa, T. T., J. MacGee, J. A. Morrison, and C. J. Glueck. 1974. Quantitative analysis of cholesterol in 5 to $20 \mu l$ of plasma. $J$. Lipid Res. 15:286-290.
26. Turley, S. D., and J. M. Dietschy. 1978. Re-evaluation of the $3 \alpha$-hydroxysteroid dehydrogenase assay for total bile acids in bile. $J$. Lipid Res. 19:924-928.

27. Lowry, O. H., N. J. Rosebrough, A. L. Farr, and R. J. Randall. 1951. Protein measurement with Folin phenol reagent. J. Biol. Chem. 193:265-275.

28. Rosenman, R. H., S. O. Byers, and M. Friedman. 1952. The mechanism responsible for the altered blood cholesterol content in deranged thyroid states. J. Clin. Endocr. Metab. 12:1287-1299.

29. Goldstein, J. L., and M. S. Brown. 1977. The low-density lipoprotein pathway and its relation to atherosclerosis. Annu. Rev. Biochem. 46:897-930.

30. Norum, K. R., A.-C. Lillieqvist, and C. A. Drevon. 1977. Coenzyme-A-dependent esterification of cholesterol in intestinal mucosa from guinea-pig. Influence of diet on the enzyme activity. Scand. J. Gastroenterol. 12:281-288.

31. Drevon, C. A. 1978. Cholesteryl ester metabolism in fat- and cholesterol/fat-fed guinea pigs. Atherosclerosis. 30:123-136.

32. Mitropoulos, K. A., S. Balasubramaniam, S. Venkatesan, and B. E. A. Reeves. 1978. On the mechanism for the regulation of 3-hydroxy-3-methylglutaryl-coenzyme A reductase, of cholesterol $7 \alpha$ hydroxylase and acyl-coenzyme A: cholesterol acyltransferase by free cholesterol. Biochim. Biophys. Acta. 530:99-111.

33. Balasubramaniam, S., K. A. Mitropoulos, and S. Venkatesan. 1978. Rat-liver acyl-CoA:cholesterol acyltransferase. Eur. J. Biochem. 90:377-383.

34. Keren, D. F., H. L. Elliott, G. D. Brown, and J. H. Yardley. 1975. Atrophy of villi with hypertrophy and hyperplasia of Paneth cells in isolated (Thiry-Vella) ileal loops in rabbits. Gastroenterology. 68:83-93.

35. Turley, S. D., and J. M. Dietschy. 1979. Regulation of biliary cholesterol output in the rat: dissociation from the rate of hepatic cholesterol synthesis, the size of the hepatic cholesteryl ester pool, and the hepatic uptake of chylomicron cholesterol. J. Lipid Res. 20:923-934.

36. Edwards, P. A., H. Muroya, and R. G. Gould. 1972. In vivo demonstration of the circadian rhythm of cholesterol biosynthesis in the liver and intestine of the rat. J. Lipid Res. 13:396-401.

37. Shefer, S., S. Hauser, V. Lapar, and E. H. Mosbach. 1972. Diurnal variation of HMG-CoA reductase activity in rat intestine. J. Lipid Res. 13:571-573.

38. Tomkins, G. M., and I. L. Chaikoff. 1952. Cholesterol synthesis by liver. I. Influence of fasting and of diet. J. Biol. Chem. 196:569-573.

39. Cayen, M. N. 1969. The effect of starvation and cholesterol feeding on intestinal cholesterol synthesis in the rat. Biochim. Biophys. Acta. 187:546-554.

40. Innerarity, T. L., and R. W. Mahley. 1978. Enhanced binding by cultured human fibroblasts of apo E-containing lipoproteins as compared with low density lipoproteins. Biochemistry. 17:1440-1447.

41. Innerarity, T. L., R. E. Pitas, and R. W. Mahley. 1980. Receptor binding of cholesterol-induced high-density lipoproteins containing predominantly apoprotein $\mathrm{E}$ to cultured fibroblasts with mutations at the low-density lipoprotein receptor locus. Biochemistry. 19:4359-4365.

42. Pittman, R. C., A. D. Attie, T. E. Carew, and D. Steinberg. 1979. Tissue sites of degradation of low density lipoprotein: application of a method for determining the fate of plasma proteins. Proc. Natl. Acad. Sci. USA. 76:5345-5349.

43. Stein, Y., G. Halperin, and O. Stein. 1981. The fate of cholesteryl linoleyl ether and cholesteryl linoleate in the intact rat after injection of biologically labeled human low density lipoprotein. Biochim. Biophys. Acta. 663:569-574. 